\title{
Design of a Dispensing CNC Device
}

\author{
P. Drgoňa ${ }^{1)}$, R. Štefún ${ }^{2)}$ and L. Štefke ${ }^{3)}$ \\ 1) Department of mechatronics and electronics, Faculty of electrical engineering, University of Zilina, Slovakia, \\ e-mail: peter.drgona@fel.uniza.sk \\ 2) Department of mechatronics and electronics, Faculty of electrical engineering, University of Zilina, Slovakia, \\ e-mail: rastislav.stefun@fel.uniza.sk \\ 3) Bel Power Solutions, Dubnica nad Váhom. Slovakia
}

\begin{abstract}
This article deals with the design and practical realization of a $\mathrm{CNC}$ device designed for the application of viscous materials, such as a thermal conducting paste. Paste is used for effective heat transfer what is essential in switched mode power supplies. However, its application on surface can be an issue. Designed CNC device serves for easier and more accurate application in production process using standard tubes.
\end{abstract}

Keywords - CNC design, CNC control, ball screw, stepper motor.

\section{INTRODUCTION}

At present, we are monitoring the growing trend of CNC devices deployment in the production process. Automation generally increases productivity. Human factor is limited, which means that products are better and more accurate, and the number of manufacturing errors is rapidly declining. Another very important advantage of automation machines is to relieve people from physically strenuous, monotonous and psychologically dysfunctional work. For this reason, requirement for the CNC dispending machine, which is the subject of this article, arises. From semiconductor converters, the heat generated by the dissipation power of the semiconductor components must be drained, in most cases by passive heat sinks. Very often during production a thermal conducting paste, which is often stored in tubes, is applied to the radiator by a piston pistol. The problem arises if the operator is not enough physically disposed to be able to apply the paste to the surface during the entire working shift. The role of the designed CNC device is therefore to automate the application process using standard tubes.

The basic requirements include:

- $X$-axis positioning: $\min .300 \mathrm{~mm}$

- $\quad Y$-axis positioning: $\min .400 \mathrm{~mm}$

- Z-axis positioning: min. $80 \mathrm{~mm}$ with manual offset adjustment

- Positioning accuracy $\pm 1 \mathrm{~mm}$ in all axes

- Self-calibration at zero point

- Ability to position $5 \mathrm{~kg}$ tube

- Extraction from standardised $1 \times 400 \mathrm{ml}$ and from $2 \times 400 \mathrm{ml}$ tube too

- Tolerance in dispensing two-part paste $<0.1 \%$

- Usage worm gear for extrusion

- Ability to perform any trajectory
- Configuration via GUI for standard PCs running MS Windows 7 or higher

- Configurable parameters: positions in $X, Y, Z$, positioning speed, adjustable amount of paste applied

\section{STATE OF THE ART}

Nowadays many CNC dispensing solutions are currently available on the market. For example, we can present professional solutions of dispense devices from Bdtronic. Beckhoff offers excellent software solutions for them. Fisnar also offers dispensing machines using worm gear instead of air pressure but only for small cartridges. Another option is modular solution. For example, Mitsubishi offers interesting PLC-based CNC solutions M70 or C70 series. However, no company on the market offered a solution that would fully satisfy our requirements. The main problem was that the devices were not able to work directly with our tubes, and the solutions offered required additional development, which increased the price dramatically. In addition, the SW configuration of the device and the programming of the correct behavior of the device in various operating situations was also required, e.g. during tube replacement. Very popular among hobby enthusiasts is Mach3. However, SW is not compatible with Windows 7 and is not open source too. Frequently used drivers for stepper motors include breakout boards for the MPS MP6500, Allegro's A4988 or TI's DRV8825. However, their power is poor for our application. That's why we decided to try to develop our own device, as cheap as possible.

\section{BLOCK DIAGRAM}

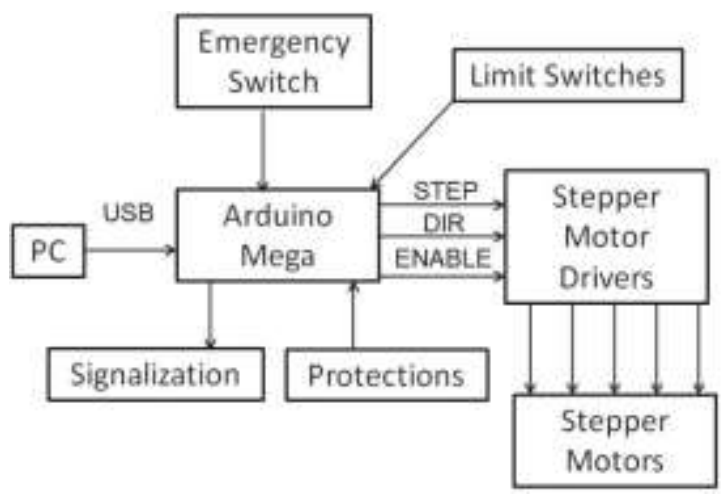

Fig. 1. Block diagram. 


\section{MECHANICAL CONSTRUCTION}

We have decided for the Compass H-1000 GS KIT from CauCau company. It is shown in Fig. 2. This is a construction with a movable portal frame that performs a working movement. On the portal, there should be a spindle, in our case a paste extrusion module (Fig. 3).

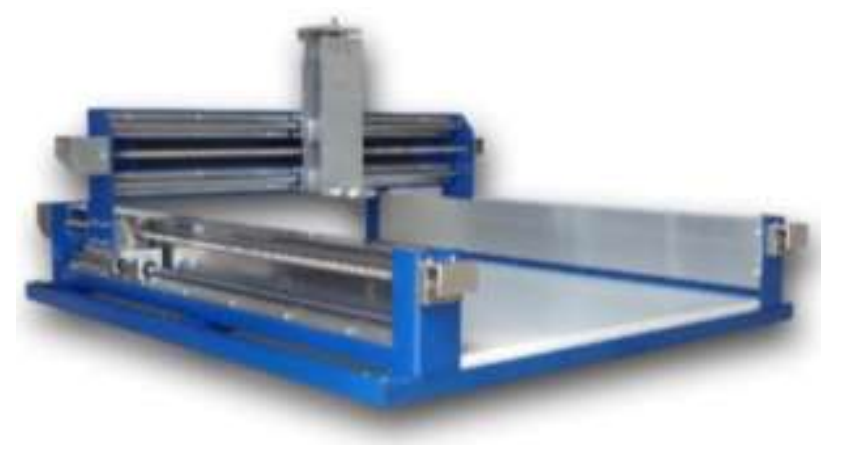

Fig. 2. Selected mechanical construction [1].

When designing a paste extrusion module, we decided to use the SBR16S. It is a system of a support rod on which there are carts with linear ball bearings with open cases. The support rod is turbid and its support is made of aluminium. The lead pair is mounted on a supporting aluminium plate. We have decided to use the ball screws to transform the rotational motion of the motor on the linear motion, because it has a higher load ability, durability, efficiency and accuracy [2], which is essential for our application.

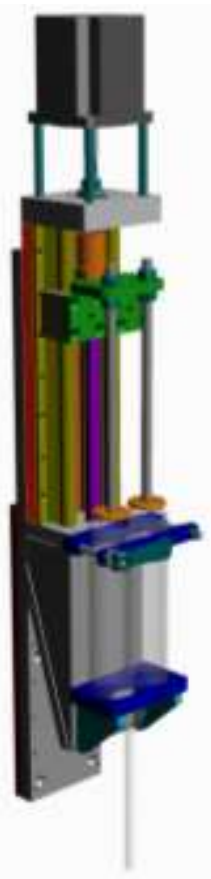

Fig. 3. 3D model of paste extrusion module.

\section{ACTUATOR SELECTION AND POWER ElECTRONICS DESIGN}

In our application, we decided to use two-phase hybrid stepper motors with bipolar winding. All motors work in the open position loop [3]. For positioning needs MotionKing motor 23HS2430 was selected, with step angle $1.8^{\circ} .4$ motors were needed, namely 2 on the $\mathrm{X}$ axis and one on the $\mathrm{Y}$ and $\mathrm{Z}$ axes.

When choosing the extruding motor, we firstly needed to know the amount of force needed to reliably push the paste out of the tube. We calculated the parameters from a pneumatic pistol used for this purpose. Working pressure is $600 \mathrm{kPa}$ at the actuator area of $5.67 \cdot 10^{-3} \mathrm{~m}^{2}$. It follows that in case of friction omission, pistol is able to develop the force according to the equation:

$$
F_{a}=p \cdot S=6 \cdot 10^{5} \cdot 5.67 \cdot 10^{-3}=3402 \mathrm{~N}
$$

The total required torque $M_{c}$, which the motor must overcome, consists of several components [4]

$$
M_{c}=M_{a c c}+M_{d}+M_{p}+M_{f}
$$

where

$M_{a c c}$ - acceleration torque [Nm],

$M_{d}$ - the torque necessary for load movement [Nm],

$M_{p}$ - torque caused by pretension of the nut [Nm],

$M_{f}$ - torque caused by friction in bearings and carts [Nm].

Torque $M_{a c c}$ directly depends on the momentum of the inertia of the system (extrusion pistons, flange, ball screw, rotor) and acceleration [5]. Since both components are low in our application, we can neglect the acceleration torque to simplify the calculation. The nut pretension is zero. We consider friction torque and it is reflected in the resulting formula as efficiency. For the final calculation of the total torque, $M_{c}$ is therefore valid [2]:

$$
M_{c}=\frac{F_{a} \cdot p \cdot o}{2000 \pi \cdot \eta}=\frac{3.4 \cdot 10^{3} \cdot 5 \cdot 0.5}{2000 \pi \cdot 0.9}=4.5 \mathrm{Nm}
$$

where $F_{a}-$ axial force $[\mathrm{N}]$, we calculated it in equation (1),

$p$ - screw thread pitch [mm],

$\eta$ - efficiency of screw drive [-], although [2] states $95 \%$, we chose $90 \%$, because we consider not always ideal conditions of the power transfer. Practically maintenance is often neglected which means that the lubrication of the bolt and nut contact will not always be ideal,

$o$ - oversizing of the entire drive by $50 \%$ recommended by [6].

Motor SX4570 from Microcon company fulfills torque requirements. Like 23HS2430 its step angle is $1.8^{\circ}$. As we can see in the Fig. 4 [6], it is possible to achieve $2.5 \mathrm{rot} / \mathrm{s}$ with enough torque at the divided step.

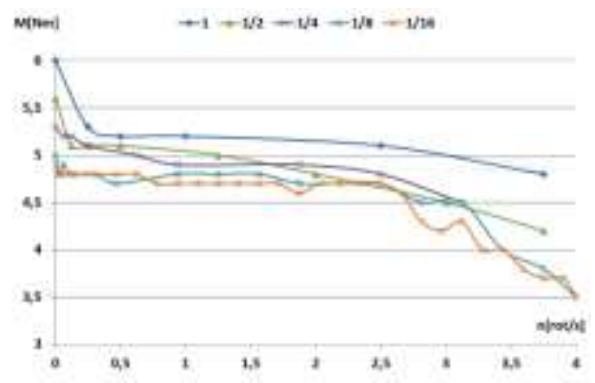

Fig. 4. Torque characteristics of motor SX4570 for current amplitude $3.8 \mathrm{~A}$ 
There are many topologies of the stepper motor driving circuits. According to [7], for maximum efficiency, we have chosen circuits with the PWM current control. We decided to use integrated circuits and therefore the IC TOSHIBA TB6600 was chosen. It provides integration of the logical circuits and two power H-bridges capable of working with a peak current up to $4.5 \mathrm{~A}$. The logical part provides sequential changes in the current reference, depending on the chosen step size division. Around this reference, the output current is switched by a hysteresis controller (Fig. 6). IC allows the stepper motor to be controlled by only three signals, namely STEP, DIR and ENABLE [8]. Rising edge of the STEP signal causes motor to move one step. Logic level of the signal DIR determines direction of motor rotation. Low level is for $\mathrm{CW}$ direction and high level is for $\mathrm{CCW}$ direction. The designed PCB allows to connect 6 stepper motors. Size of the step angle is possible to divide up to $1 / 16$ using the DIP switch. Digital isolators ISO722x isolate apart the power and logic parts of the circuit [9]. As power supply, it was used FNP300-1024, which provides $24 \mathrm{~V}$ for the power part and $12 \mathrm{~V}$ for logic part [10]. Using the voltage regulator LP2950CDT-5.0 we lowered $12 \mathrm{~V}$ to the TTL level, which is suitable for TB6600.

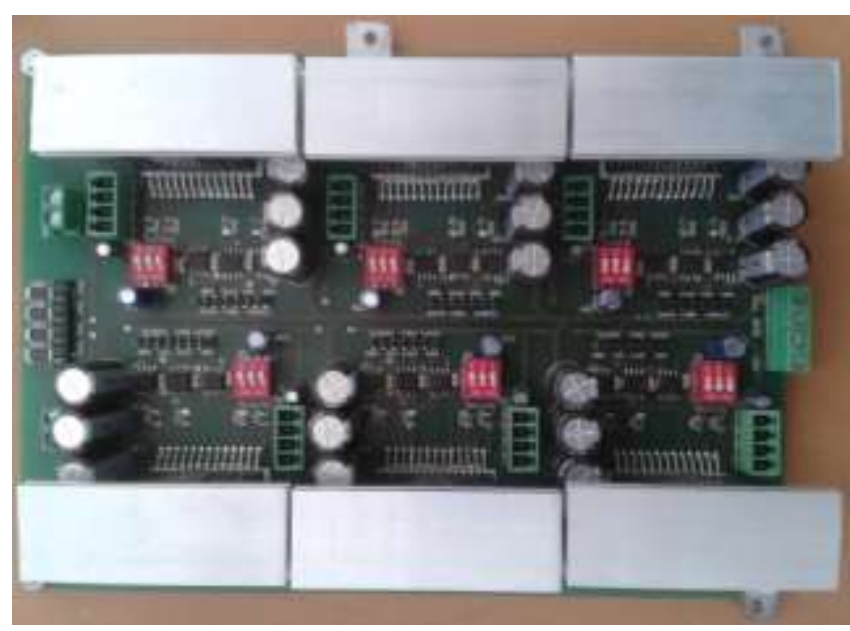

Fig. 5. Mounted PCB, top view.

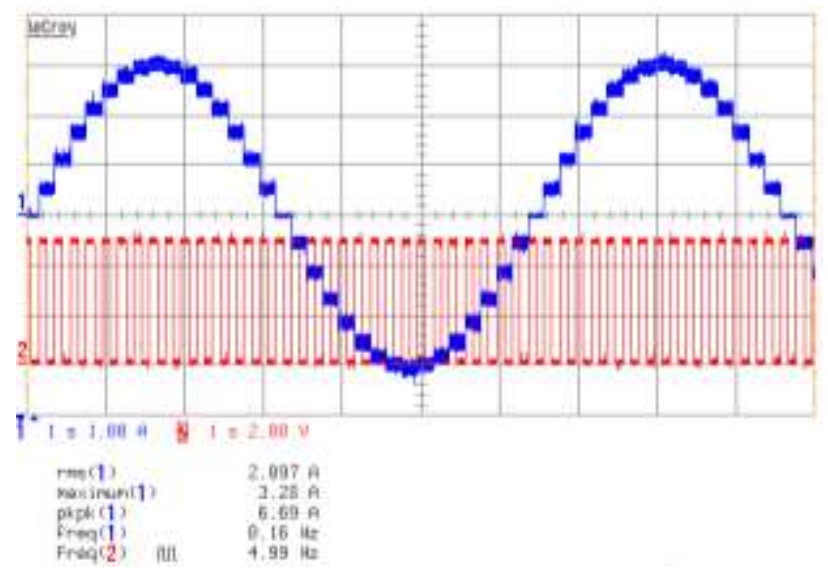

Fig. 6. Phase current of motor 23 HS 2430 for $1 / 8$ step (blue) and signal STEP (red).

\section{SELECTION OF CONTROL ELECTRONICS AND FIRMWARE}

As can be seen in Fig. 1 choice of the control electronics fell on the Arduino Mega based on ATMega 2560, which is 8-bit microprocessor from Atmel. Because we are unable to debug program using the boot loader, we used the AVR Dragon board. This requires the OCD feature that the ATMega2560 meets. As a base for the control program, we chose the open source firmware Repetier, made for 3D printers, as we needed to configure the device behavior [12].

In Fig. 7 the simplified Repetier flow chart is shown. It operates in an infinite loop. After start of the program all registers and GPIOs are set. Then communication with PC can start. Commands are in the ISO code language. It is standard for the $\mathrm{CNC}$ machines. If no command is sent to processor, then flags, interrupts, temperature of hot end or state of communication are controlled. But if the command is present in the communication buffer, the processor decides whether it is a moving instruction or instruction for setting some parameters or options. At the moving instruction processor calculates amount of steps to reach the desired point in space according to the desired velocity and acceleration. Logical level of the DIR signal and other attributes are also set. Timer makes appropriate PWM signal for motor control. After move finish the processor reads the serial communication link again.

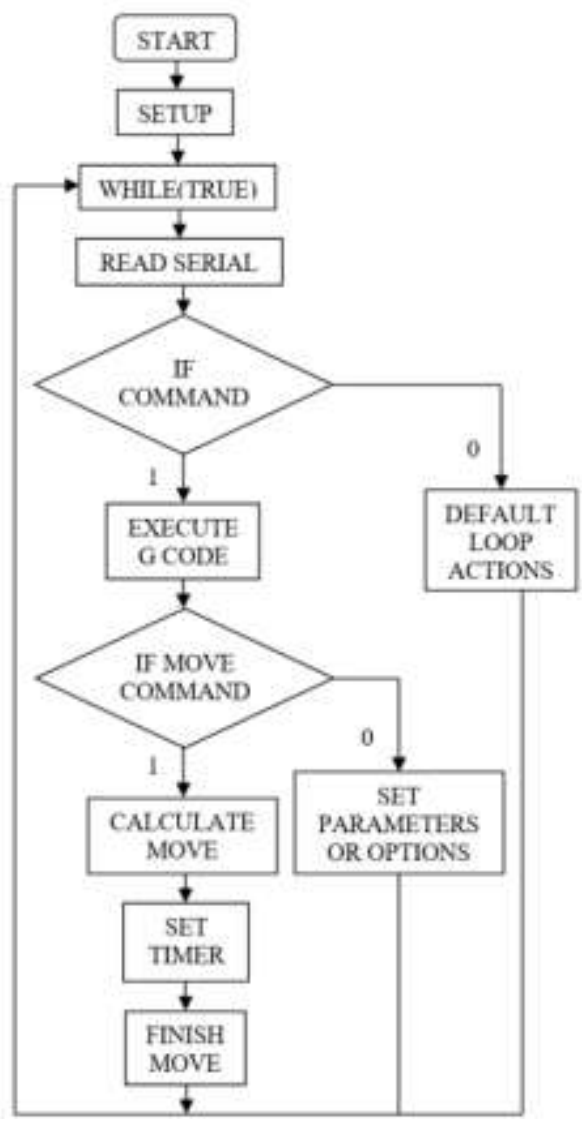

Fig. 7. Simplified Repetier flow chart. 
Because of industrial application we had to implement security features. We connected the emergency switch to the Reset pin. Pressing it will bring logical 0, which will cause processor reset and immediate shutdown of the device.

Overheating of the circuit is solved through interrupt. When the chip temperature reaches $160{ }^{\circ} \mathrm{C}$, IC switches the ALERT signal from log. 1 to log.0. The falling edge causes interrupt. In service routine all ENABLE signals first switch to log.0. Subsequently, the green LED on the signalling device goes off and the red signal starts lighting to indicate the fault condition. Before the program remains stuck in an infinite loop, the GUI application will show message that overheating has occurred. Since interrupts are used to communicate with $\mathrm{PC}$, they firstly must be enabled. The ATmel processors automatically disable all interrupts when the program is in the service routine of one of them. After the GUI message all interrupts are again disallowed with the cli() command. The only way to get a device from an endless loop is to reset the processor after cooling of drivers.

The role of the infrared gate is to tell the processor that a foreign body, most often a part of the human body is in the working area of the machine. The processor must respond with an immediate break of the motion activity. Engines must be stopped throughout the presence of the body in the detected area. We tried to use an interrupt but then there was a loss of steps, so we had to change method where the Bresenham interpolation algorithm is calculated. Return value of this method is the value of the comparator register for the timer. The output compare register changes for each step, depending on which axis performs the movement to reach the desired point in space. It also depends on the speed and acceleration required. The timer then generates the appropriate STEP signals for the individual motors to be rotated with respect to the required acceleration ramps by changing the pulse width. The current calculated ISO code line is pointed by the pointer named as cur. Its private attributes include initial speed, desired speed, acceleration, accelerator steps, and so on. The Bresenham algorithm calculates some of its attributes, mainly the difference and deviation, but also the direction. Some of these attributes change with each step, so this method is called by the processor when one of the motors moves and recalculates the attributes again and therefore we performed the implementation here. So before processing the next step, the processor checks the logical level on the input from the gate. If the log. 1 level is there, the red LED goes on, the green goes off, and the processor executes blank "nop" instructions until the logic level changes again to log.0. All motors are stopped at that moment. After the logical level change, the green LED lights up again and the red goes off. Then the attributes to which the pointer points are reset by the command cur = NULL and then recalculated, when the program is resumed. This has resulted in a ramp increase of the rotation speed, which prevents the steps being lost.

When the device operates, it may happen that the tube is emptied. Therefore, we also implemented algorithm for this case. In the method with the Bresenham algorithm, it is monitored whether or not a switch is activated. The switch is activated when the push pistons reach the lower position. If so, the attributes pointed by the 'cur' pointer are reset and the red LED lights up, and the green goes off. In the GUI is then printed "Change_Tub" message.
Current values of the STEP, DIR and ENABLE signals of the extruder are stored in auxiliary variables. Subsequently, the ENABLE signal is set to $\log .1$ as well as the DIR signal. In for cycle it is generated rising edge of the STEP signal until the pistons reach the top position. Next switch gives the processor information that the pistons are in the upper position. Consequently, it is necessary to change the level of the logical signal DIR back to log.0 to be able to move the pistons downwards. Then the device waits for the human operator to replace the tube. The operator sets the pistons to position where the paste starts to flow from the mixer by pushing the button. If everything is ready to continue the application process, operator presses the OK button. Next, for cycle, which generates rising edge of the STEP signal, stops and the LED lights switch to the original combination. The STEP, DIR, and ENABLE signals are rewritten to the original logical values. By doing so, the tube replacement algorithm is terminated and the device can continue its work.

\section{PRACTICAL REALIZATION AND EVALUATION OF ACHIEVED RESULTS}

We attached paste extrusion module onto the $Z$-axis aluminium plate of the positioning mechanism. For the connection of the selected motors to the structure, we used spacers in combination with flexible couplings. To control the actuators, we used the proposed PCB. It works reliably. The positioning motors are operated on a 1/8 step at a current amplitude $3.3 \mathrm{~A}$. For a more fluent tube extrusion, we chose for the SX4570 1/16 step at a current amplitude $3.8 \mathrm{~A}$.

We placed the wires in the energy chains to prevent them from being twisted. The demonstration of the application over the circular trajectory is shown in Fig. 7. As far as the specification in Part I is concerned, we have successfully fulfilled all the requirements.
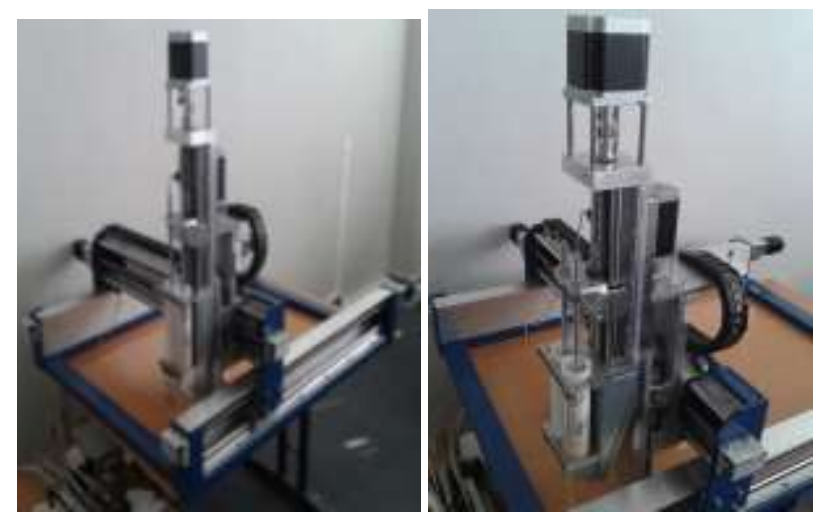

Fig. 8. Practical realization of the $\mathrm{CNC}$ device.

Ability of the manual $Z$ axis offset adjustment is possible thanks to an appropriate design. The change range is $364.5 \mathrm{~mm}$.

The overall positioning accuracy is given by the precision of the stepper motor, ball screw, nut and by backlash of coupling. Maximum deviation was measured 
$\pm 0.04 \mathrm{~mm}$, so we did not exceed required accuracy \pm 0.1 $\mathrm{mm}$.

TABLE I.

REACHED DIMENSIONS OF THE WORKSPACE AND MAXIMUM VALUES OF AXES SPEEDS AT THE SELECTED ACCELERATION $500 \mathrm{Mm} / \mathrm{s}^{2}$

\begin{tabular}{|c|c|c|c|}
\hline Axis & $\begin{array}{c}\text { Required } \\
\text { dimension } \\
{[\mathbf{m m}]}\end{array}$ & $\begin{array}{c}\text { Reached } \\
\text { dimension } \\
{[\mathbf{m m}]}\end{array}$ & $\begin{array}{c}\text { Maximum } \\
\text { reached speed } \\
{[\mathbf{m m} / \mathbf{s}]}\end{array}$ \\
\hline $\mathrm{X}$ & 300 & 665 & 185 \\
\hline $\mathrm{Y}$ & 400 & 570 & 242.5 \\
\hline $\mathrm{Z}$ & 80 & 80 & 51 \\
\hline
\end{tabular}

Calibration at the zero point was achieved by using limit switches on each axis. During tests, we were able to reliably position $5 \mathrm{~kg}$ of weight. When designing a paste extrusion module, we used a worm gear with accentuate on making possible to rework the module as easily as possible for use both one- and two-part tubes. During application of a two-part paste, both pistons are set in one plane and driven by the same motor, so there is no deviation in application. By selecting the appropriate firmware, we have determined that the device is able to perform any trajectory. Whether it is a straight line or a circle. Thanks to the Repetier Host GUI application, compatible with Windows 7 and higher, we are able to easily configure all the required parameters. These are the positions in the $X, Y, Z$ axes as well as the position of the motor for extrusion purposes, the speed and acceleration of the drives. It is also possible to show the path in the application. The amount of applied paste can be adjusted to a certain level by changing the speed of the extrusion motor.

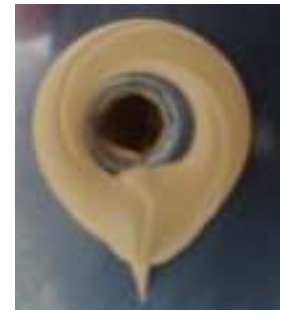

Fig. 9. Result of paste application along the circular path

\section{CONCLUSION}

We have built a functional device capable of applying viscous materials according to the specified specification. We have selected mechanical construction on which we have attached the designed module for the paste extrusion. Selected motors are controlled by the open source firmware which was improved according to our needs. The control algorithm for tube exchange and some safety features were added. The device will serve to automate the application process in the production process. For the future, the safety must be improved more, the device will have to be placed in the cage and the only accessible area will be covered with an infrared gate. It is also necessary to program the device so that a lot of paste is not dispensed during application of the material.

\section{ACKNOWLEDGMENT}

Authors would like to thanks to APVV-15-0571Research of the optimum energy flow control in the electric vehicle system, KEGA 073ŽU-4/2017Implementation of modern tools for teaching automotive electronics and electromobility, Vega - 0479/17- Research of optimal methods of energy transfer control in systems with accumulating elements and Bel Power Solutions, Dubnica nad Váhom.

\section{REFERENCES}

[1] P. Stefanec, M. Paskala, B. Dobrucky et al., "Control System for Preheating of Molds with using PLC," in International Conference on Industrial Technology and Management Science (ITMS 2015). Book Series: ACSR-Advances in Comptuer Science Research, vol. 34, pp. 1647-1652.

[2] Frantisek Duchon, Peter Hubinsky, Jaroslav Hanzel et al., "Intelligent vehicles as the robotic applications," in: 5th International Conference on Modelling of Mechanical and Mechatronics Systems (MMaMS) 2012 - MODELLING OF MECHANICAL AND MECHATRONICS SYSTEMS. Book Series: Procedia Engineering, vol. 48,pp. 105-114. https://doi.org/10.1016/j.proeng.2012.09.492

[3] http://www.cnc1.eu/sk/KompasH1000GSKIT.htm [cited 11. March 2017]

[4] http://www.thomsonlinear.com/downloads/screws/Leadscrews_Ba 1lscrews_Splines_cten.pdf [cited 14. April 2017]

[5] Tomas Urica, Anna Simonova, Slavomir Kascak, "Control design and simulation of continuous systems," in Electric power engineering EPE 2017 18th international scientific conference, IEEE, 2017, pp. 125-129. ISBN 978-1-5090-6405-2. https://doi.org/10.1109/EPE.2017.7967263

[6] http://www.linearmotiontips.com/calculate-motor-drive-torqueball-screws/ [cited 10. may 2017]

[7] Andrej Tereň, Pavol Špánik, Ivan Feňo, "DC/DC converters with soft (ZVS) switching," in ELEKTRO 2001: section: electrical engineering. Žilina: University of Žilina, pp. 82-87. ISBN 807100-836-2.

[8] Viliam Fedak, Frantisek Durovsky, Robert Uveges et al., "HIL Simulator of Drives of an Industrial Robot with 6 DOF," in ELEKTRONIKA IR ELEKTROTECHNIKA, vol. 21, issue 2, pp. 14-19. Published 2015.

[9] http://microcon.cz/ [cited 10. may 2017]

[10] Anna Simonová, Libor Hargaš, Dušan Koniar, Zuzana Loncová, and Miroslav Hrianka, "Mathematical analysis of complete operation cycle of a system with two-position controller," in ELEKTRO 2016 - 11th international conference IEEE 2016, pp. 213-215. ISBN 978-1-4673-8698-2 - CD-ROM. https://doi.org/10.1109/ELEKTRO.2016.7512067

[11] TOSHIBA, DATASHEET TB6600, www.toshiba.com

[12] O. Hock, P. Drgona, and M. Paskala, „Simulation Model of Adjustable Arm Using Denavit-Hartenberg Parameters," in ELEKTRO 10th International Conference, 2014, pp. 176-179. ISBN 978-1-4799-3721-9.

https://doi.org/10.1109/ELEKTRO.2014.6847896 